\title{
How Can Neuroscientists Respond to the Climate Emergency?
}

\author{
Adam R. Aron, ${ }^{1, *}$ Richard B. Ivry, ${ }^{2}$ Kate J. Jeffery, ${ }^{3}$ Russell A. Poldrack, ${ }^{4}$ Robert Schmidt, ${ }^{5}$ Christopher Summerfield, ${ }^{6, *}$ \\ and Anne E. Urai ${ }^{7}$ \\ ${ }^{1}$ Department of Psychology and Neuroscience Graduate Program, University of California San Diego, San Diego, USA \\ ${ }^{2}$ Department of Psychology, University of California, Berkeley, USA \\ ${ }^{3}$ Division of Psychology and Language Sciences, University College London, UK \\ ${ }^{4}$ Department of Psychology, Stanford University, Stanford, CA, USA \\ ${ }^{5}$ Department of Psychology, University of Sheffield, Sheffield, UK \\ ${ }^{6}$ Department of Experimental Psychology, University of Oxford, Oxford, UK \\ ${ }^{7}$ Cold Spring Harbor Laboratory, Cold Spring Harbor, NY, USA \\ *Correspondence: adamaron@ucsd.edu (A.R.A.), christopher.summerfield@psy.ox.ac.uk (C.S.) \\ https://doi.org/10.1016/j.neuron.2020.02.019
}

The world faces a climate emergency. Here, we consider the actions that can be taken by neuroscientists to tackle climate change. We encourage neuroscientists to put emissions reductions at the center of their everyday professional activities.

\section{Introduction}

Human activity has heated the planet by $\sim 1.1^{\circ} \mathrm{C}$ since pre-industrial times, and the average global temperature seems to be growing exponentially ( $\mathrm{Xu}$ et al., 2018). The international consensus body of climate scientists, backed by governments, has called for a reduction in emissions of $\sim 50 \%$ by 2030 to try to keep the temperature increase to $1.5^{\circ} \mathrm{C}$ (IPCC, 2018). A $50 \%$ reduction in emissions is a daunting undertaking that will require us to completely restructure our global economy around renewable sources of energy and sustainable development. If temperature is allowed to go much beyond $2^{\circ} \mathrm{C}$ as business-as-usual projections suggest (Rogelj et al., 2016), then the possible consequences include mass despeciation, periodic deadly temperatures, rising sea levels that jeopardize many current population centers, food and water insecurity, ever-rising poverty levels, and the possibility of mass migration and further armed conflict between nations (Wallace-Wells, 2019)

In this NeuroView, our focus is on the actions that can be taken by neuroscientists-researchers, clinicians, educators, administrators, and students - who make up the readership of Neuron. Our goal here is to ask how neuroscientists can reduce the emissions and waste that arise as a consequence of professional activities, including flying, teaching, research, and administration (Favaro, 2014). In the face of political inaction, it is easy to feel that individuals count for little and that the cause is hopeless. However, many earlier movements have shown us that when individuals work together to take collective nonviolent action, the consequences can ripple and undergird major societal and policy change (Chenoweth et al., 2011). Although climate science, social change, and geopolitics are not the traditional focus of our field, we believe that neuroscientists have important roles to play in shaping our generation's obligation to tackle the climate emergency.

\section{Flying Less}

A major way we can reduce our emissions as a field is by flying less (Nathans and Sterling, 2016). Air travel accounts for around $4 \%$ of $\mathrm{CO}_{2}$ emissions globally (IPCC, 2018) and is slated to grow dramatically over coming years. These emissions are mostly caused by a small percentage of the world's population that includes many academics. The international nature of academic work has normalized frequent air travel as an intrinsic part of being a successful scientist, and many neuroscientists travel tens of thousands of miles per year to give talks or posters at invited seminars, workshops, and conferences. A researcher traveling from London to San Diego on an economy class return ticket would produce about 2.6 tons of $\mathrm{CO}_{2}$. According to some estimates, these $\mathrm{CO}_{2}$ emissions from one return flight are equivalent to living without a car for a year (2.4 tons), nearly double the emissions associated with switching a household to entirely renewable energy (1.4 tons), and nearly 3 times the emissions associated with moving from an omnivorous to a fully plant-based diet for a year (0.8 tons) (Wynes and Nicholas, 2017); these emissions are also 13 times the annual per capita emissions of a person in Tanzania. The carbon footprint of many researchers is thus disproportionately large compared to the population average. Furthermore, the largest conference in our field, the Society for Neuroscience meeting, takes place in a US city each year and attracts over 30,000 attendees. Given that most delegates arrive by air, including many from outside the US, it is easy to estimate that this conference alone contributes tens of thousands of tons of $\mathrm{CO}_{2}$ in flights alone.

\section{Carbon Offsets}

One common argument against reducing $\mathrm{CO}_{2}$ emissions is that rather than flying less, we should simply pay for carbon offsets when purchasing air tickets. Carbon offsets are voluntary payments toward schemes that aim to reduce emissions or even reverse them ("drawdown") and thus to neutralize the impact of our consumption. For example, one major neuroscience conference now offers a link for people to pay $\$ 15$ to offset one ton of $\mathrm{CO}_{2}$, with that money used to buy water filters in Honduras to reduce the 
need for local people to use wood when boiling drinking water.

However, a closer look at carbon offsets suggests that they are at best a partial solution and at worst actively harmful. There are at least three serious arguments against offsets. The first concerns uncertainty about the benefit that the offsets actually confer. It is important to realize that offsets only truly neutralize the impact of consumption if the schemes meet the additionality requirement; i.e., the emissions reductions would not have happened without our payment. For example, if a government scheme began to sanitize Honduran drinking water, then the offset one paid for one's flight would not be additional. Analysis of a major scheme, the Clean Development Mechanism under the Kyoto protocol, showed that the vast majority of projects for emissions reduction were not truly additional and also noted that the same problem continues to bedevil current schemes (Haya et al., 2019) There is thus uncertainty about whether such schemes truly offset the $\mathrm{CO}_{2}$ emitted by flying.

Second, the scale of the activity required to truly offset the emissions cost of flying may be unfeasible or prohibitive. For example, reforestation enhances net uptake of carbon from the atmosphere and is thus widely regarded to be an important tool in combating climate change. However, reforestation may actually increase carbon emissions if not managed carefully (Naudts et al., 2016). Furthermore, each tree planted only offsets $\sim 20$ kilos of $\mathrm{CO}_{2}$ per year, whereas annual emissions from aviation are about of half a billion tons; thus, vast, continent-sized stretches of land would need to be devoted to reforestation for viable offsetting (Skidmore et al., 2019).

The final argument is that some offset schemes may inadvertently be actively harmful. Some may create perverse incentives. For example, methane capture schemes funded from offsets have been found to increase the profitability of coal mines, thus potentially prolonging their lifespan ${ }^{6}$. More generally, offset schemes may be actively detrimental to reducing emissions if they stem the tide of people who reduce their flying by offering a false hope that their footprint can be reduced with a small tax. In short, offset schemes may discourage us from flying less when in actuality, flying less is required to avoid catastrophic climate change. There is thus a risk that offsets do more harm than good.

Additionally, it may be naive to imagine that a small payment, such as $\$ 15$ per ton, can offset the several tons of emissions typically generated by a long-haul return flight. For comparison, the approximate cost of direct air carbon capture-a truly additional scheme-is $\sim \$ 200$ per ton (Keith et al., 2018), an order of magnitude higher. And indeed, the consequence of paying the substantial fee for this nearcertain and additional benefit would be to actually reduce flying itself. Overall, these considerations suggest that by far the best solution is to fly less and thus to help ensure that the planet's reserves of carbon remain locked in the ground for as long as possible.

\section{Climate Justice}

Frequent travel allows neuroscientists to promote their work and thus increases the rate at which new ideas spread. Travel also builds and fosters our international community, as friendships, connections, and collaborations are forged between individuals in geographically distant laboratories with shared research interests. International travel may be particularly beneficial to more junior researchers or those from the developing world, who have not yet had a chance to establish themselves within the neuroscience community. Any call to climate action must therefore seek to mitigate the risk that some members will be disproportionately disadvantaged by steps taken to reduce emissions.

The term "climate justice" recognizes that those whose actions have contributed the least to our planetary predicament thus far (i.e., the poor, the young, and the vulnerable) are likely to suffer the worst consequences of the climate emergency. Conversely, this means that those senior researchers who have built their careers by establishing networks of collaborations through many decades of frequent air travel should be first in line to commit to flying less. They should also be on the front line of arguing for institutional change in the service of emissions reductions.

More generally, the culture of the academic neuroscience community currently places junior researchers (as well as those from developing countries) in an inescapable bind-it is very difficult to establish oneself without committing to traveling internationally to take up training positions abroad, to meet relevant senior colleagues, and to present work at conferences and seminars. We need to rethink how we build our networks within the neuroscience community and to normalize other modes of teaching, collaboration, and information dissemination that do not rely on in-person meetings. We need to find ways to allow everyone in the community-including students and early career researchersto prosper without being obliged to commit to frequent travel.

Of note, widespread adoption of new modes of interaction (such as immersive virtual reality) that do not require travel will have the additional benefit of widening participation to groups who typically find travel more difficult-for example, those from countries subject to visa restrictions or for whom travel is prohibitively expensive, those with disabilities, or those with young children or other family responsibilities that make prolonged absence from home particularly difficult.

\section{Virtual Participation at Conferences and Seminars}

The organizers of conferences and workshops should consider measures that will reduce the carbon footprint of the event while continuing to widen participation. First, virtual participation can be encouraged. Although this has historically been a rather clumsy option to implement, rapid technological development means that immersive systems allowing for fluent interactions are now available at relatively low cost. One simple but effective measure is to make talks and submissions (e.g., papers, posters) freely available so that interested delegates from further afield do not have to travel by air to participate. Some conferences already offer excellent prototypes for this model. For example, the popular machine-learning conference NeurIPS makes videos of all events immediately and freely available online in an easily accessible format.

Another suggestion is to organize the meeting according to a "hub and spokes" scheme where the main meeting (e.g., in country A, the "hub") is accompanied by 
smaller meetings (in countries B-Z, the "spokes") that are attended by local participants, with hub and spokes meetings linked by videoconference. The "spokes" meetings can combine broadcasts of talks and elaborate on discussions from the main meeting but simultaneously provide local activities that permit networking and facilitate the building of collaborations closer to home. Neuroscientists can also organize, support, and attend more local conferences and workshops. Although this may not completely replace international meetings, it is a step forward in reducing their number and size. More generally, we hope that it will become standard practice for conferences to publish their estimated carbon footprint (according to an agreed standard) to allow potential delegates to take this into consideration when choosing which meeting to attend (Ponette-González and Byrnes, 2011).

Academics also frequently travel to deliver seminars. Organizers who are populating the speaker program could consider offering the option of a virtual visit, whereby the talk is delivered remotely and beforehand while the $Q$ \& A and/or 1:1 meetings could occur live via videoconference; good examples already exist. This approach would permit most of the high-quality interaction that normally occurs during a research visit. If the organizers are looking for in-person visitors, in the spirit of climate justice, they might consider reserving places for early career researchers or those from under-represented groups who stand to benefit more from the opportunity to meet and socialize face-to-face with like-minded colleagues. In addition, host institutions should ensure that in those areas where train travel is a viable alternative (e.g., across Europe), speaker invitations could include an encouragement to travel by land and confirm that the associated costs would be reimbursed even if they are higher than for plane travel. Funders have a part to play here, by making up the difference between flying and other forms of travel.

\section{Reducing Waste and Energy} Consumption in Our Research Scientific research can be an enormously resource-intensive exercise. Even seemingly innocuous activities, such as using the internet, can contribute. The information and communications technology sector, which underpins much research, is estimated to be responsible for about $2 \%$ of global $\mathrm{CO}_{2}$ emissions. Most neuroscience researchers will recognize the energy-intensive nature of their research, in particular if it involves the analysis of large datasets or large-scale computational simulations. Although not directly related to climate change, we should also take seriously the ecological effects of plastic waste from laboratory equipment, protective clothing, animal care, and bench consumables, as well as toxic chemicals, all of which threaten biodiversity. We are also permanently depriving future generations of non-renewable resources like helium. As a profession, we need to work toward more sustainable practices in the long term.

Some researchers have begun initiatives to try and reduce waste (see http:// www.mygreenlab.org; for an example, see Rae, 2019). However, the institutions within which we work must take on board the seriousness of the environmental crisis and weigh it equally with a commitment to individual health and safety. Funders and institutions need to consider whether all research is essential, given the planetary destruction it entails. One approach could be for institutional auditing of the resources used to generate a research output. Currently, people are rewarded for the money they spend (bigger grants meaning more promotion, etc.), but not penalized in any way for what they consume in the course of their research. We need a culture change within and across our institutions to tackle the climate emergency. A spin-off benefit will be allowing small, low-resource-use labs to compete on a more equal footing with their giant counterparts.

\section{Using Our Positions of \\ Responsibility to Tackle the Climate Emergency}

Neuroscientists are researchers, clinicians, educators, and administrators and also public voices for science-based policies. As researchers and clinicians, we can fly less and try to influence others to do the same. As educators, we can also seek to directly instill in our staff and students a sense of urgency about the climate emergency through our personal interactions and in formal teaching settings. For example, we can teach classes that foreground the climate emergency or else build climate into our standard syllabus, for example, using the climate emergency when teaching about cognitive biases, decision-making, programming, or big data (Aron, 2019). As neuroscientists, we can collectively try to build a lab culture that acknowledges the scale of the challenge posed by the climate emergency and that is supportive of local initiatives that are geared at raising awareness or protesting inaction. One could convene or request a lab meeting on how the team can reduce emissions in its everyday activities.

As administrators and members of institutional bodies, we have yet more leverage. For example, we may sit on department and university-wide committees that make decisions about travel, spending, and investments. We can recommend that videoconferencing is used for seminars; that large open-house visits are replaced with systematic virtual interviews; that catering for departmental events has mainly vegetarian or vegan options; that our departments and institutions shift funds out of the principal banks that are heavily implicated in funding fossil fuel extraction; that our university endowments and pensions are divested of fossil fuel stocks; that we and our colleagues learn how to purge our personal retirement funds of fossil fuel stocks; that our campuses shift local energy supplies away from natural gas to majority electricity and eventually renewable-supplied electricity; that our campuses invest in better transportation to discourage personal solitary driving; and many other actions. Students and early career researchers who do not sit on institutional committees can nevertheless organize themselves to campaign for these changes, especially where departments are slow to react. Finally, we can also be public voices for science-based responses to the climate emergency.

\section{How Can Funders of Neuroscience Research Contribute?}

The neuroscience community also encompasses people involved in grant review and the disbursement of research funds. They too have a significant role in tackling the climate emergency. When 
researchers submit requests for research funds to government agencies or charitable foundations, they are required to provide a detailed breakdown of the financial costs of the research. These are scrutinized by reviewers and grant panels, and funds can be withheld if costs seem unwarranted. We suggest that funding bodies request an emissions-counting component, calculated according to agreed guidelines. For example, grants that propose a large number of international flights or that invoke major power costs (e.g., fMRI scanning or heavy computing resources) should be asked to note (and/or justify where useful) the emissions. These costs may be non-negligible. For example, annual emissions from a single fMRI scanner may be as much as 16 tons of $\mathrm{CO}_{2}$, even without taking into account substantial manufacturing and decommisioning contributions (Herrmann and Rock, 2012). Of note, the intention here is not to debar researchers from engaging in valuable research activities or essential travel but merely to encourage a culture in which we meticulously count the cost of research to the climate emergency.

Funding bodies have tremendous influence and thus a great opportunity to tackle this issue. One potential model is the Athena Swan Charter, which sought to promote equality of opportunity among the sexes in UK higher education. Since 2011, a Silver Award, offered to department and universities with an outstanding track record of promoting gender equality under the Athena Swan commission, has been a precondition for eligibility for funding from major UK sources. In the years immediately following this change, the number of university departments with awards increased from just seven to over 200. In a similar vein, funding bodies who are evaluating large-scale institutional bids for research centers, infrastructure improvements, or doctoral training programs could consider the emissions reduction commitments of the applicant as a part of the evaluation process or even consider restricting eligibility to de- partments or institutes that have met appropriate targets. These steps may seem unnecessarily burdensome for academics who may already be overworked. However, we argue that a radical problem calls for radical solutions. These specific suggestions aside, we hope that funders will consider using their influence wisely to tackle the climate emergency.

\section{Conclusions}

Emissions reductions must happen urgently. While large political systems equivocate, grassroots action can sway political activity. The actions proposed here are thus important steps toward prompting the major top-down social and economic changes that must eventually happen, such as equitable carbon taxes and widespread investment in renewable energy sources. Neuroscientists can be part of this change. They can make personal changes such as flying less, and by doing so and advertising it, they will support a growing culture that has the potential to lead to major emissions reductions. Neuroscientists are also situated within universities, professional societies, and research bodies where they can work with colleagues to inculcate myriad changes, including virtual conferencing, measuring and reducing waste, divesting banking and investments from exposure to fossil fuel companies, and reforming funding practices. In so doing, they can contribute to a fundamental shift in the ethos of our institutions to put emissions reductions into the very fabric of standard operations at every level. Doing so will safeguard the ongoing practice of neuroscience. We hope that the readers of Neuron will feel inspired to take up the challenges we raise here and that we can work together to ensure that the mysteries of the brain will still be studied with equal vigor 100 years from now.

\section{ACKNOWLEDGMENTS}

Thanks to Parke Wilde and Eran Mukamel for helpful comments on the manuscript.

\section{REFERENCES}

Aron, A.R. (2019). The Climate Crisis Needs Attention from Cognitive Scientists. Trends Cogn. Sci. 23, 903-906.

Chenoweth, E., Stephan, M.J., and Stephan, M.J (2011). Why civil resistance works: The strategic logic of nonviolent conflict (Columbia University Press).

Favaro, B. (2014). A carbon code of conduct for science. Science 344, 1461.

Haya, B., Cullenward, D., Strong, A.L., Grubert, E., Heilmayr, R., Sivas, D., and Wara, M. (2019). Managing Uncertainty in Carbon Offsets: Insights from California's Standardized Approach. Stanford Law School ENRLP Program Working Paper https://law.stanford.edu/publications/managinguncertainty-in-carbon-offsets-insights-from-californiasstandardized-approach/.

Herrmann, C., and Rock, A. (2012). Magnetic resonance equipment (MRI) - Study on the potential for environmental improvement by the aspect of energy efficiency. PE International AG, Report http://large.stanford.edu/courses/ 2012/ph240/nam2/docs/herrmann.pdf.

IPCC (2018). Intergovernmental Panel on Climate Change Report: Global Warming of $1.5^{\circ} \mathrm{C}$. https://www.ipcc.ch/sr15/.

Keith, D.W., Holmes, G., St. Angelo, D., and Heidel, K. (2018). A process for capturing CO2 from the atmosphere. Joule 2, 1573-1594.

Nathans, J., and Sterling, P. (2016). How scientists can reduce their carbon footprint. eLife 5, e15928.

Naudts, K., Chen, Y., McGrath, M.J., Ryder, J. Valade, A., Otto, J., and Luyssaert, S. (2016). Europe's forest management did not mitigate climate warming. Science 351, 597-600.

Ponette-González, A.G., and Byrnes, J.E. (2011) Sustainable Science? Reducing the Carbon Impact of Scientific Mega-Meetings. EBL 2, 65-71.

Rae, C. (2019). Climate change. https://www sussex.ac.uk/psychology/abc-lab/climate-change.

Rogelj, J., den Elzen, M., Höhne, N., Fransen, T. Fekete, H., Winkler, H., Schaeffer, R., Sha, F. Riahi, K., and Meinshausen, M. (2016). Paris Agreement climate proposals need a boost to keep warming well below $2{ }^{\circ} \mathrm{C}$. Nature 534 , 631-639.

Skidmore, A.K., Wang, T., de Bie, K., and Pilesjö, P. (2019). Comment on "The global tree restoration potential". Science 366, 6469 .

Wallace-Wells, D. (2019). The Uninhabitable Earth: A Story of the Future (Allen Lane).

Wynes, S., and Nicholas, K. (2017). The climate mitigation gap: education and government recommendations miss the most effective individual actions. Environ. Res. 12, 074024.

Xu, Y., Ramanathan, V., and Victor, D.G. (2018) Global warming will happen faster than we think. Nature 564, 30-32. 